\title{
Library Event Matching event classification algorithm for electron neutrino interactions in the NOvA detectors
}

\author{
C. Backhouse, R. B. Patterson
}

Lauritsen Laboratory, California Institute of Technology, Pasadena, California 91125, USA

\begin{abstract}
We describe the Library Event Matching classification algorithm implemented for use in the NOvA $v_{\mu} \rightarrow v_{e}$ oscillation measurement. Library Event Matching, developed in a different form by the earlier MINOS experiment, is a powerful approach in which input trial events are compared to a large library of simulated events to find those that best match the input event. A key feature of the algorithm is that the comparisons are based on all the information available in the event, as opposed to higher-level derived quantities. The final event classifier is formed by examining the details of the best-matched library events. We discuss the concept, definition, optimization, and broader applications of the algorithm as implemented here. Library Event Matching is well-suited to the monolithic, segmented detectors of $\mathrm{NO} v \mathrm{~A}$ and thus provides a powerful technique for event discrimination.
\end{abstract}

Keywords: library matching, classification algorithm, particle identification, NOvA

\section{Introduction}

Classifying images into a small number of categories is a common task in scientific and industrial fields. In particle physics, this task usually involves interpreting particle detector data to determine the type of particles, interactions, or decays present. Given the sheer volume of information that can be collected, the data is often first reduced to a set of derived quantities by running algorithms that pull out key features: clusters, tracks, showers, jets, etc. While this form of lossy compression is acceptable in some applications, it is worth exploring whether a classification scheme that uses all of the available information is feasible, even in cases where the data volume is high.

In this article we describe such a classification scheme developed to categorize neutrino scattering events recorded in the NOvA detectors. In the Library Event Matching (LEM) algorithm, a trial event of unknown type is compared to a large number of known "library" events to find those events that are most similar to the trial event. The properties of those best-matched library events reveal the likely nature of the trial event. A distinguishing feature of LEM is that the comparisons are made using the energy depositions directly, to avoid any information loss from calculating higher-level variables. This fundamental phi- ${ }^{32}$ losophy of LEM was developed within the MINOS collabora- ${ }^{33}$ tion for its own neutrino event categorization needs [1, 2, 3, 4]. ${ }^{34}$ The LEM version described in this article has substantial differ- ${ }^{35}$ ences from its predecessor, many of which are motivated by the ${ }^{36}$ higher spatial resolution of the NOvA detectors.

While we use $\mathrm{NO} v \mathrm{~A}$ as our case study, the approach dis- ${ }_{37}$ cussed is generalizable and could be usefully applied to any highly segmented detector, from hadron calorimeters determin- 38 ing jet multiplicity to cubic kilometer arrays collecting neutri- ${ }_{39}$

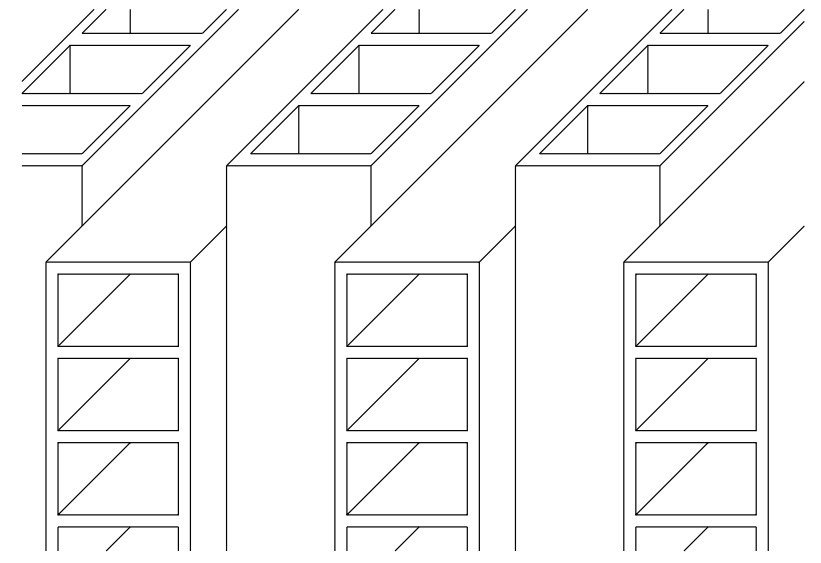

Figure 1: A sketch of the structure of the NOvA detectors. $4 \mathrm{~cm} \times 6 \mathrm{~cm}$ cells run the length of each $16 \mathrm{~m} \times 16 \mathrm{~m}$ plane. The alternating vertical and horizontal orientations can be seen. They are filled with liquid scintillator and each contains a looped wavelength-shifting fiber (not shown), as described in the text. This cut-away sketch is diagrammatic only. The real cells have rounded corners and the ends of the cells are capped for instrumentation and oil containment purposes. The neutrino beam is incident from the left.

nos from astrophysical sources. As with many machine learning algorithms, LEM requires a large number of known examples from each classification category. In particle physics applications, these would typically come either from an advanced Monte Carlo simulation or from calibration sources.

\section{The NOvA experiment}

The NOvA (NuMI Off-axis $v_{e}$ Appearance) experiment studies the phenomenon of neutrino flavor oscillation [5]. Neutrinos 

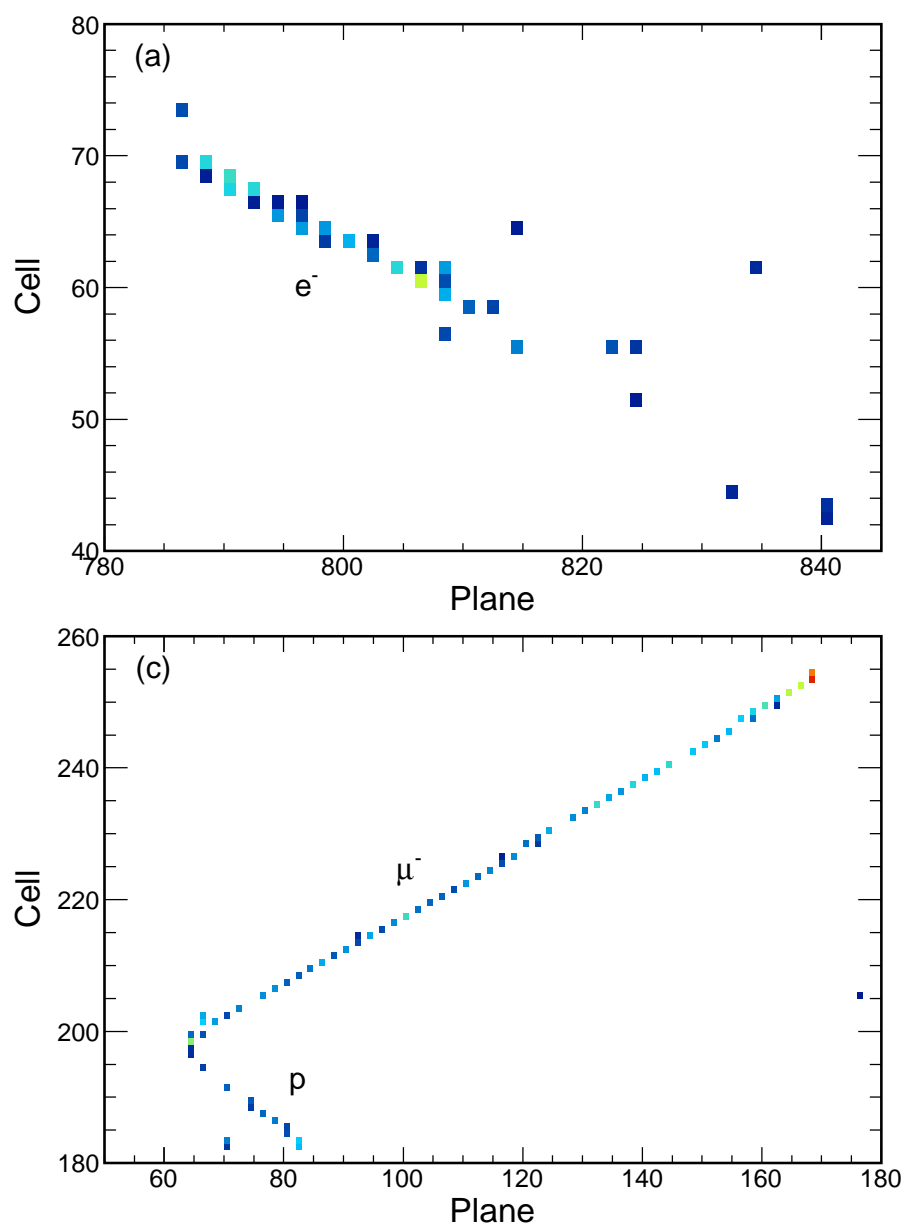

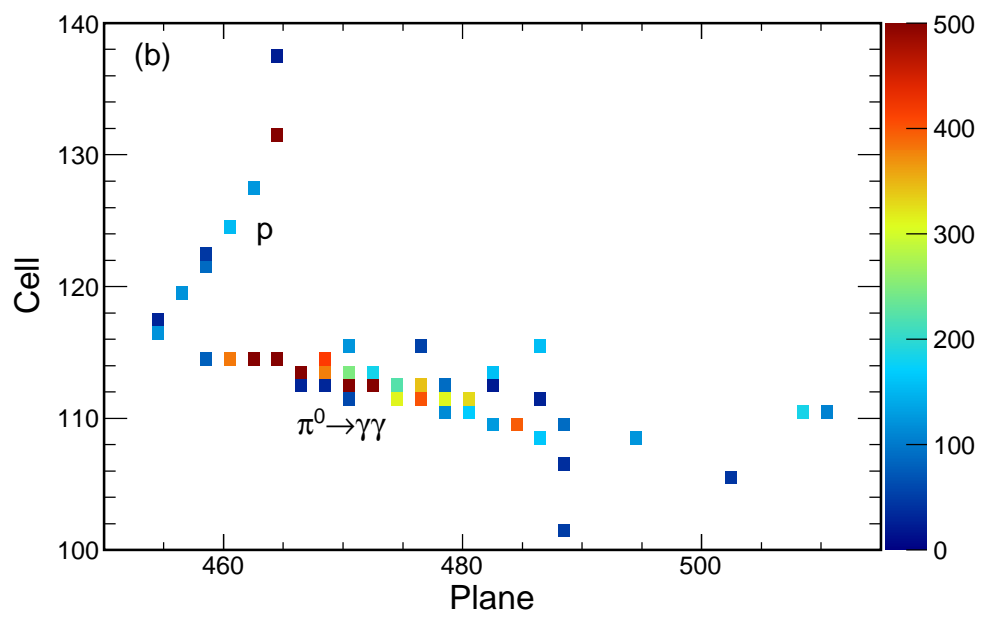

Figure 2: Example simulated events in the $\mathrm{NO} v \mathrm{~A}$ detectors. Only one of the two views is shown in each case. Each box represents one cell and is positioned according to its plane number (horizontal axis) and cell number (vertical axis). The color scale indicates the charge deposited in photoelectrons, and is common to all three panels. (a) A $v_{e} \mathrm{CC}$ event, with the electron-induced electromagnetic shower clearly visible. (b) A neutral current event with a $\pi$. The upper track is due to a proton. This event shows that the two showers from $\pi^{0} \rightarrow \gamma \gamma$ are not always distinct. (c) A $v_{\mu} \mathrm{CC}$ event, with the usual tell-tale long, straight muon track. Note that the axis ranges are approximately doubled for this panel relative to the first two.

neutrino's flavor. In the 1 to $3 \mathrm{GeV}$ energy range of $\mathrm{NO} v \mathrm{~A}$, this electron will be accompanied, with similar probabilities, by a proton (quasi-elastic scattering), a nucleon plus a pion (resonant scattering), or a richer hadronic shower (deep inelastic scattering). While nuclear effects blur these crisp definitions, these three scattering types are useful for conveying the variety of shapes that signal events in $\mathrm{NO} v \mathrm{~A}$ can take. The $\sim 1 \mathrm{GeV}$ electron in the final state produces an electromagnetic shower in the detector that has a width of a few cells and runs longitudinally an average distance of $2.5 \mathrm{~m}$ ( 40 planes). Figure 2a shows a simulated $v_{e} \mathrm{CC}$ interaction in the $\mathrm{NO} v \mathrm{~A}$ Far Detector.

The primary mis-identification background comes from neutral-current (NC) interactions, particularly those where the recoil hadronic system contains a $\pi^{0}$. The $\pi^{0}$ decays quickly to two photons, each of which induces an electromagnetic shower that is essentially indistinguishable from an electron-induced shower. NC $\pi^{0}$ events, taken as a whole, look sufficiently different from signal $v_{e}$ CC events that we can reject them well, but the differences are sometimes obscured:

- The presence of two electromagnetic showers, rather than one, can reveal a $\pi^{0}$ in the final state. However, if one of the showers has low energy or overlaps the other in the detector, it can be missed.

- Photon-induced showers are separated from the neutrino interaction point due to the distance traveled by the photon prior to its conversion. This gap is a tell-tale sign of 


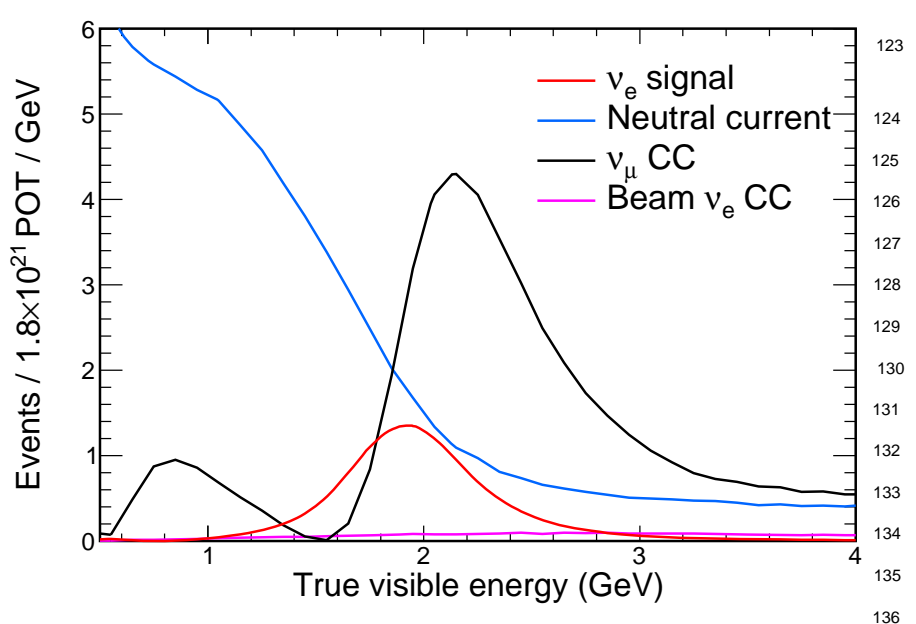

Figure 3: Signal and background distributions of visible energy expected in the Far Detector sample. The effect of neutrino oscillations is included. Visiblez7 energy is defined as the incident neutrino energy except in the case of neutral current events where the outgoing neutrino energy is subtracted. The $v_{e}$ sig- $^{138}$ nal to be identified by LEM is shown in red. The neutral current, $v_{\mu}$ charged 139 current, and intrinsic beam $v_{e}$ charged current components are blue, black, and ${ }_{40}$ magenta respectively. a photon, but in some cases the gap will be too small to resolve. The conversion length in $\mathrm{NO} v \mathrm{~A}$ is $50 \mathrm{~cm}$.

- Photon-induced showers begin with two particles (an elec-147 tron/positron pair) rather than one, but these cases can end ${ }_{148}$ up indistinguishable given the energy resolution of the de-149 tector. ing leads to reconstructed energies lower than those of sig- ${ }^{153}$ nal events. However, interactions from a sufficiently high- ${ }^{154}$ energy neutrino or with a large energy transfer can fall in $^{155}$ the signal region of 1 to $3 \mathrm{GeV}$ reconstructed energy.

Figure $2 \mathrm{~b}$ shows a simulated NC event with a $\pi^{0}$.

Additional background comes from $v_{\mu} \mathrm{CC}$ scattering, which ${ }^{158}$ produces a muon in the final state. The muon leaves a long track of activity in the detector with a characteristic energy deposition per unit pathlength. These are readily removed from the sample ${ }_{160}$ due to the clear muon track except in cases where the muon is low in energy or is lost amongst other activity. In these cases, ${ }_{162}$ the background is similar to $\mathrm{NC}$ interactions, with neutral pions ${ }_{163}$ playing the same role. Figure 2c shows a $v_{\mu} \mathrm{CC}$ example.

The NuMI beam also includes a $2 \%$ contamination of $v_{e+65}$ These $v_{e}$ interact identically to the $v_{e}$ from oscillations and ${ }_{166}$ thus constitute a background to the $v_{\mu} \rightarrow v_{e}$ oscillation measure- ${ }_{167}$ ment. However, their rate is low and their energies are some- ${ }_{168}$ what higher. Figure 3 illustrates the energy differences among ${ }_{169}$ all the event classes before any selection cuts have been applied..$_{170}$

Since the $v_{e}$ CC signal falls within a known energy range, 171 we can safely remove lower and higher energy events up front. 172 For all figures and tables that follow, we require events to have 173 reconstructed visible energies between $0.5 \mathrm{GeV}$ and $4 \mathrm{GeV}$. $\quad{ }_{174}$

\section{Library Event Matching concept}

At the heart of the LEM algorithm is the comparison of each unknown trial event to a large number of known library events, with the comparisons based on low-level information collected by the detector. For $\mathrm{NO} v \mathrm{~A}$, this means using the calibrated energy depositions in all the detector cells directly rather than forming higher-level objects such as showers and tracks from those.

Once the very best matches are found (here, the best $0.0001 \%$ of all library events), their known properties are used to estimate the properties of the trial event. In the simplest version of LEM, the fraction of the best matches that are signal events can be used as the discriminant. Appendix A.1 discusses the relationship between LEM and other machine learning techniques.

\subsection{The matching metric: motivation}

When comparing two events, a metric is needed to quantify how similar they are. It is instructive to look at the MINOS case briefly, as the situation there is somewhat simpler $[1,2,3,4]$.

The MINOS detector has a segmented structure analogous to that of the $\mathrm{NO} v \mathrm{~A}$ detector, but the effective spatial resolution for events of interest is significantly lower. A $v_{e} \mathrm{CC}$ signal event in MINOS involves only a couple dozen active "strips" (the analogue of NOvA's cells), and these active strips are clustered in a relatively compact pattern. Thus, two events with the same underlying particle kinematics have a good chance of having identical (or near-identical) arrangements of active strips. The readout electronics report the number of photoelectrons detected in each active strip. Since this charge measurement suffers from shot noise (typical charge: $\sim 8$ photoelectrons), strips with identical energy depositions may report different charges. The level of difference is governed by Poisson statistics.

These details guided the form of matching metric used by MINOS, which can be thought of as the likelihood $\mathcal{L}$ that the two events' recorded charges represent the same underlying energy depositions:

$$
\log \mathcal{L}=\sum_{i}^{\text {strips }} \log \left[\int P\left(a_{i} \mid \lambda\right) P\left(b_{i} \mid \lambda\right) d \lambda\right],
$$

where $a_{i}$ is number of photoelectrons registered by the $i^{\text {th }}$ strip of event $\mathrm{A}, b_{i}$ is the same for event $\mathrm{B}, P(n \mid \lambda)$ is the Poisson probability of observing $n$ given mean $\lambda$, and the sum runs over all strips active in at least one of the events. A higher $\log \mathcal{L}$ for a pair of events means a better match. Before $\mathcal{L}$ is calculated, the events, which in general occur in different parts of the detector, are spatially aligned by shifting them so that their charge-weighted mean strip positions, rounded to the nearest strip, overlap.

In the MINOS metric $\mathcal{L}$, displaced energy depositions in the two events do not get their charges directly compared. To obtain good matches for a trial event, the library must be large enough to span minor variations in active strip positions for nominally equivalent events. This is possible in MINOS given the limited spatial resolution of the detectors for $v_{e} \mathrm{CC}$ events. That is, the library can be expected to give reasonable coverage of all 
possibilities. Requiring exact charge agreement across the $\sim 20_{222}$ active strips, though, would be combinatorically overwhelming. 223 The Poisson factors take care of this, with acceptably different 224 charges able to contribute appropriately to the match score. 225

The NO $v$ A detectors are significantly more finely-grained than those of MINOS. This makes event discrimination easier in principle since more details are visible, but it makes the above matching metric impractical. It is much less likely that "equivalent" activity in the trial and library events will fall on the same cells. What is needed is a matching metric that rewards activity in nearby cells without requiring them to lie directly on top of one another. A library event identical to the trial event should 226 still be a perfect match, but events with similar charges offset ${ }_{227}$ by a cell or so should still score well.

The metric we use draws its motivation from electrostatics.229 Two Coulomb charge distributions of similar shape, but with $_{230}$ opposite signs, will have a low electrostatic potential energy ${ }_{23}$ when overlaid and examined together, as the attraction between the opposite signed charges counters the internal repulsion of the like-signed charges. Two overlaid charge distributions with ${ }^{232}$ dissimilar shape suffer the internal repulsion but lack the benefit ${ }_{233}$ of mutual attraction, leading to a large potential energy. Given ${ }_{234}$ the electrostatic analogue to what follows, we use "energy" to $\mathrm{o}_{235}$ refer to the LEM match score for the remainder of the article ${ }_{236}$ unless otherwise stated. Lower energies correspond to better matches.

The match energy is defined as

$$
E=E_{A}+E_{B}+E_{A B},
$$

where $E_{A}$ is the self-energy (repulsion) of event A's charges, $E_{B^{241}}$ is the self-energy of event $\mathrm{B}$ 's charges, and $E_{A B}$ is the (negative) ${ }^{242}$ energy due to the the $\mathrm{A} / \mathrm{B}$ attraction. The charges are taken to be the recorded energy depositions in the NO $v \mathrm{~A}$ cells. Treating ${ }^{243}$ the electrostatic analogue as exact for a moment, the self-energy terms are given by

$$
E_{A}=\frac{1}{2} \sum_{i j}^{\text {cells }} \frac{a_{i} a_{j}}{r_{i j}}, \quad E_{B}=\frac{1}{2} \sum_{i j}^{\text {cells }} \frac{b_{i} b_{j}}{r_{i j}},
$$

with $a_{i}\left(b_{i}\right)$ the recorded deposition in the $i^{\text {th }}$ cell of event A (event B) and with $r_{i j}$ the distance between cells $i$ and $j$. The $r_{i j}=0$ case is handled again with an electrostatic analogue by distributing all charges uniformly across their individual cells.

(See Appendix A.2.)

The interaction term is given by

$$
E_{A B}=-\sum_{i j}^{\text {cells }} \frac{a_{i} b_{j}}{r_{i j}} .
$$

Before evaluating this sum, the events are globally aligned with one another according to a separately reconstructed interaction vertex. $^{1}$

A perfect match, in which events A and B have identical depositions in identical cell positions, would yield $E=0$. A poorly

\footnotetext{
${ }^{1}$ Alignment by charge-weighted mean cell position was also studied and gives similar classification performance.
}

matched pair with charges far away from one another will have large energy $E \approx E_{A}+E_{B}$.

Eq. (4) can be recast in terms of one set of charges embedded in the field of the other:

$$
\begin{aligned}
E_{A B} & =-\sum_{i}^{\text {cells }} a_{i} V_{i} \\
\text { where } V_{i} & =\sum_{j}^{\text {cells }} \frac{b_{i j}}{r_{i j}} .
\end{aligned}
$$

The advantage of this formulation is that $V$ can be precalculated for each trial event, along with the self-energies of the trial and library events. When matching against a large number of library events using (5), the complexity is linear in the number of charges rather than requiring a double sum over both trial and library charges.

\subsection{The matching metric in NOvA}

While the NOvA matching metric is inspired by electrostatics, there is no reason to expect that the precise form above will yield the best sensitivity. We incorporate the following generalizations.

- Above, $r_{i j}$ is calculated as the Euclidean distance in terms of the number of planes $\Delta p_{i j}$ and number of cells $\Delta c_{i j}$. However, $\mathrm{NO} v \mathrm{~A}$ events are boosted forward and cover many planes longitudinally but relatively few cells transversely, so we assign different relative importance to separations in the two directions.

- The $r^{-1}$ falloff with distance is generalized to $r^{-\alpha}$.

- The importance of larger charges relative to smaller ones is adjusted by raising all charges to a power $\beta$.

The resulting form of the matching metric still follows Eq. (2), but the self-energy and interaction terms are now given by

$$
\begin{aligned}
E_{A} & =\frac{1}{2} \sum_{i j}^{\text {cells }} a_{i}^{\beta} T_{i j} a_{j}^{\beta}, \quad E_{B}=\frac{1}{2} \sum_{i j}^{\text {cells }} b_{i}^{\beta} T_{i j} b_{j}^{\beta} \\
E_{A B} & =-\sum_{i}^{\text {cells }} a_{i}^{\beta} U_{i}
\end{aligned}
$$

with the transfer matrix $T_{i j}$ and field $U_{i}$ given by

$$
\begin{aligned}
T_{i j} & =\left(\frac{\Delta p_{i j}^{2}}{\sigma_{p}^{2}}+\frac{\Delta c_{i j}^{2}}{\sigma_{c}^{2}}\right)^{-\alpha / 2} \\
U_{i} & =\sum_{j}^{\text {cells }} T_{i j} b_{j}^{\beta} .
\end{aligned}
$$

The electrostatics version is recovered by setting

$$
\sigma_{p}=\sigma_{c}=\alpha=\beta=1 .
$$




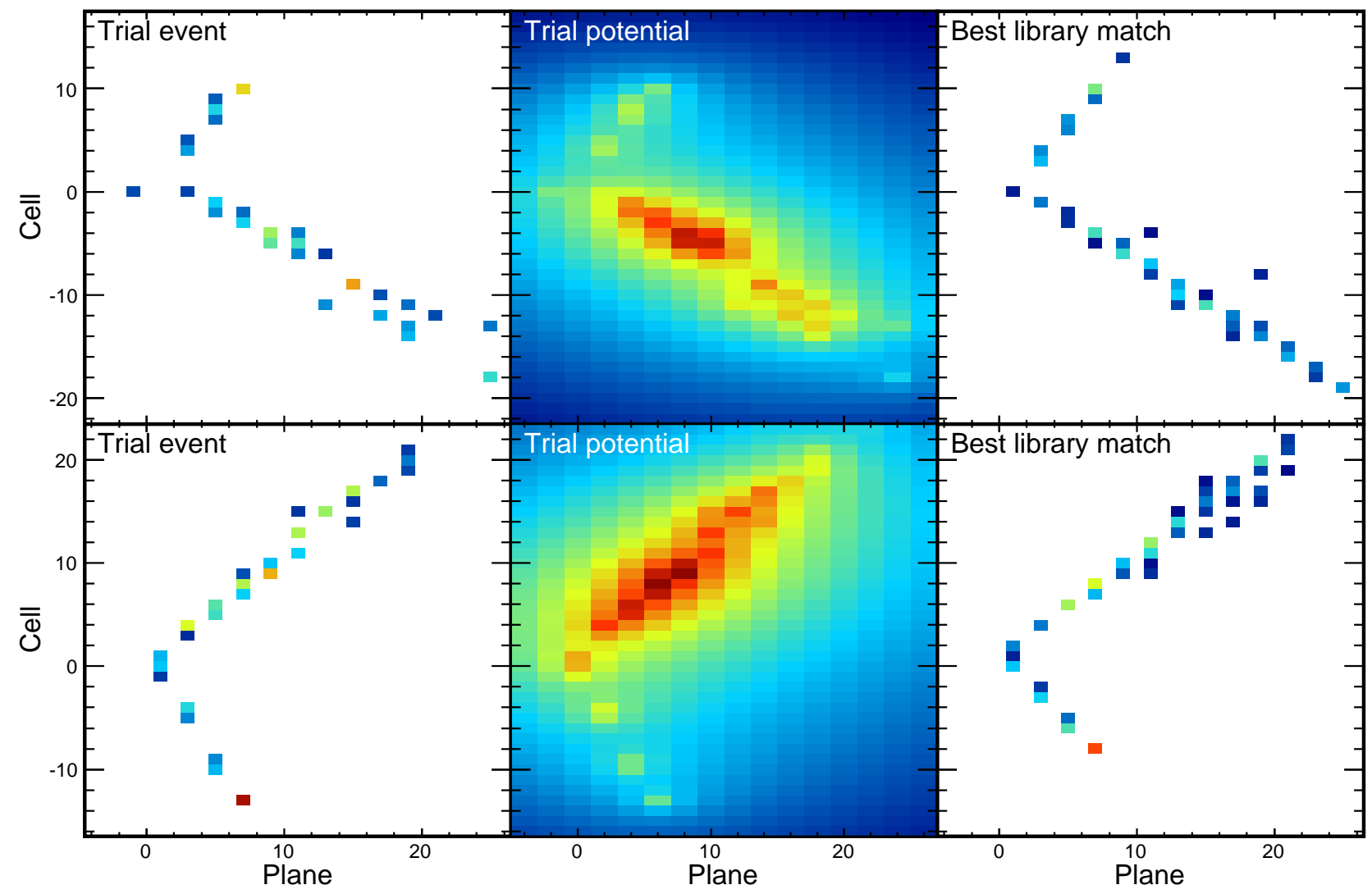

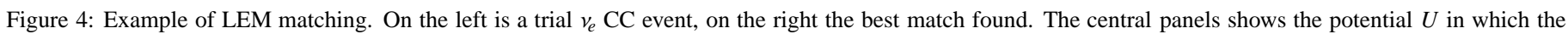
library events are placed in order to calculate the match energy. The upper panels show one view, and the lower panels show the other.

We ran toy experiments with different values of these parame-268 ters and calculated a figure-of-merit for each to optimize per-269 formance. The parameters chosen were:

$$
\begin{aligned}
\sigma_{p} & =0.286 \\
\sigma_{c} & =0.095 \\
\alpha & =0.25 \\
\beta & =0.5 .
\end{aligned}
$$

The first two parameters validate the intuition that transverse differences should be considered more significant than longitu- ${ }^{278}$ dinal ones. The third parameter specifies a $1 / \sqrt[4]{r}$ falloff with distance, slower than the electrostatic analogue. For $\beta$, note ${ }^{280}$ that the simple presence or absence of activity in a cell conveys information regardless of its charge. Having $0<\beta<1$ moves ${ }^{282}$ the metric towards this binary "on/off" interpretation and away ${ }^{283}$ from a charge-proportional weighting.

\section{The library}

The library consists of $77 \mathrm{M}$ simulated neutrino events, of ${ }_{290}$ which $18 \mathrm{M}$ are signal $v_{e}$ CC events, $29 \mathrm{M}$ are background $v_{\mu 291}$ $\mathrm{CC}$ and $\mathrm{NC}$ events, and $30 \mathrm{M}$ are $\pi^{0}$-enriched $\mathrm{NC}$ background events. Each trial event that LEM classifies is compared to these $77 \mathrm{M}$ events to find the 1,000 library events that are most sim- ilar to it, as quantified by the metric above. ${ }^{2}$ Figure 4 shows an example trial event along with its event potential $U$ and its best-matched library event.

The library events are generated ahead of time using the full NOvA Monte Carlo simulation chain including realistic neutrino flux, cross sections, and detector components. The flux is calculated using a FLUKA/FLUGG implementation of the beamline elements [7], the neutrino interactions are simulated by GENIE [8], and particle propagation through the detector geometry is handled by GEANT4 [9]. Simulated energy depositions in the liquid scintillator are converted into expected signals by $\mathrm{NO} v \mathrm{~A}$ electronics and data acquisition simulation code. The registered signals are corrected for light attenuation in the cells' fibers using standard $\mathrm{NO} v \mathrm{~A}$ calibration procedures.

$\mathrm{NC}$ events containing neutral pions are the dominant misidentification background owing to the electromagnetic showers from $\pi^{0} \rightarrow \gamma \gamma$. Thus, we supplement the base background library sample with a $\pi^{0}$-enriched library sample. To build this enriched sample, we apply a cut that selects out only those neutral current events with a $\pi^{0}$ present in the final state as reported by GENIE.

The library events are generated according to the expected $v_{\mu}$ flux (for background) or a 100\% $v_{\mu} \rightarrow v_{e}$ transmutation (for signal), without regard to any actual probabilities for neutrino

\footnotetext{
${ }^{2}$ This statement is modified in Sec. 7.1 when we discuss speed optimizations.
} 
flavor change. Oscillations are introduced into the library later ${ }_{343}$ by event weighting. This is discussed in Sec. 5 below. Ap- 344 pendix A.3 describes the oscillation probabilities used.

While increasing the library size beyond the $77 \mathrm{M}$ events 346 would provide incremental improvement in classification performance, we observe that these gains enter logarithmically with the number of library events once the library is sufficiently large. In an earlier version of the algorithm, we found that doubling the library size provided only $1 \%$ gain in physics sensi- ${ }^{347}$ tivity. In light of the computational requirements discussed in $^{348}$ Section 7, additional library events are not worthwhile for our ${ }^{349}$ application.

\subsection{Event flipping}

To good approximation, flipping an event transversely in one or both views produces an equally valid event. We use such 353 flipping to effectively quadruple the size of the library when the 354 matching is performed. Each library event is used in each of the 355 four possible configurations, and the best of the four is retained. 356 This symmetry is not quite perfect in the $\mathrm{NO} v \mathrm{~A}$ detectors. At-357 tenuation in the readout fibers leads to subtly different charge 358 resolutions and threshold effects on transversely opposing sides 359 of an event, and NuMI neutrinos at the Far Detector enter at a $3^{\circ}$ upwards angle. Nevertheless, the best-scoring matches come 360 from the four possible flipped configurations with nearly equal probability: $26 \%$ from unflipped events, $50 \%$ from events with either one of the two views flipped, and $24 \%$ from events with ${ }^{361}$ both views flipped.

\section{Decision tree}

As library size increases, the fraction of an event's bes ${ }^{365}$ matches that are truly signal tends toward the probability that ${ }^{366}$ the trial event itself is signal. Further, all of the information ${ }^{367}$ available in the trial event is used when determining this prob- ${ }^{-368}$ ability. It is in this sense that LEM is optimal.

For a library of finite and practical size, though, this signal ${ }^{370}$ fraction alone does not contain the full information extractable. ${ }^{371}$ Other statistics constructed from the details of the best matches may, for example, indicate that the matches are drawn from an 372 area of sparse library coverage and are thus less reliable. The most powerful approach given a finite library is to construct several statistics describing the matches and to feed these into ${ }^{373}$ one of the standard multivariate analysis techniques to extract374 the final classifier. In LEM, five variables are constructed from 375 the 1,000 best library matches and are used as inputs to a deci-376 sion tree, along with the calorimetric energy of the trial event 377 as a sixth input.

\subsection{Weighted fraction of signal matches}

The basic quantity measuring what fraction of the best matches are signal events can be improved upon by weighting up the truly best matches over the lesser ones when calculating ${ }_{38}$ the signal fraction. We use the weighting

$$
w_{n}^{\prime}=\exp \left(-\lambda\left(\frac{E_{n}}{E_{1000}}\right)^{\gamma}\right)
$$

where $n$ is the match index, $E_{n}$ is the energy of the $n^{\text {th }}$ best match for the trial event, and $E_{1000}$ is the energy of the final $\left(1000^{\text {th }}\right)$ best match. The optimized values used for $\lambda$ and $\gamma$ in $\mathrm{NO} v \mathrm{~A}$ are

$$
\begin{aligned}
\lambda & =6.67 \\
\gamma & =10 .
\end{aligned}
$$

The typical ratio of weights $w_{1000}^{\prime} / w_{1}^{\prime}$ is $\sim 0.1 \%$, indicating that the most important matches are captured within the first thousand.

In practice, the weight must also include the oscillation probabilities alluded to earlier:

$$
w_{n}=w_{n}^{\prime} P_{n}^{\mathrm{osc}},
$$

where $P_{n}^{\text {osc }}$ is the oscillation probability of match $n$, as described in Appendix A.3.

All sums below that are indexed by $n$ run over the match list. For notational convenience we also define $W \equiv \sum_{n} w_{n}$. This weighting scheme is used for all five quantities formed from the best-match list. The first is the weighted fraction of signal matches,

$$
f_{\text {sig }}=\frac{1}{W} \sum_{n, \text { sig }} w_{n}
$$

where this sum includes only those terms due to signal matches.

\subsection{Mean hadronic y}

Signal events in which the outgoing electron carries only a small fraction of the incident neutrino's energy will look very much like NC background events. The kinematic quantity $y$ (or rather, $1-y)$ measures this fraction: $1-y=K_{e} / K_{v}$, where we've used $K_{e}$ and $K_{v}$ as the outgoing and incoming lepton energies to avoid confusion with the match energies $E$. If a trial event matches well to signal events with high $y$, this can suggest that the trial event is in fact a high- $y$ NC event. A second input is the mean $y$ for the best matches:

$$
\langle y\rangle=\frac{1}{W} \sum_{n} w_{n} y_{n} .
$$

\subsection{Mean matched charge fraction}

Matched charge fraction is an independent measure of the quality of the library matches, separate from the match energy. For each trial/match pair, this is the quantity of charge that has a counterpart on identical cells in the two events divided by the total charge in the two events:

$$
f_{Q}=\frac{2 \sum_{i}^{\text {cells }} \min \left(a_{i}, b_{i}\right)}{\sum_{i}^{\text {cells }}\left(a_{i}+b_{i}\right)} .
$$

The weighted average of the matched charge fraction over all the matches yields the next input:

$$
\left\langle f_{Q}\right\rangle=\frac{1}{W} \sum_{n} w_{n} f_{Q, n} .
$$



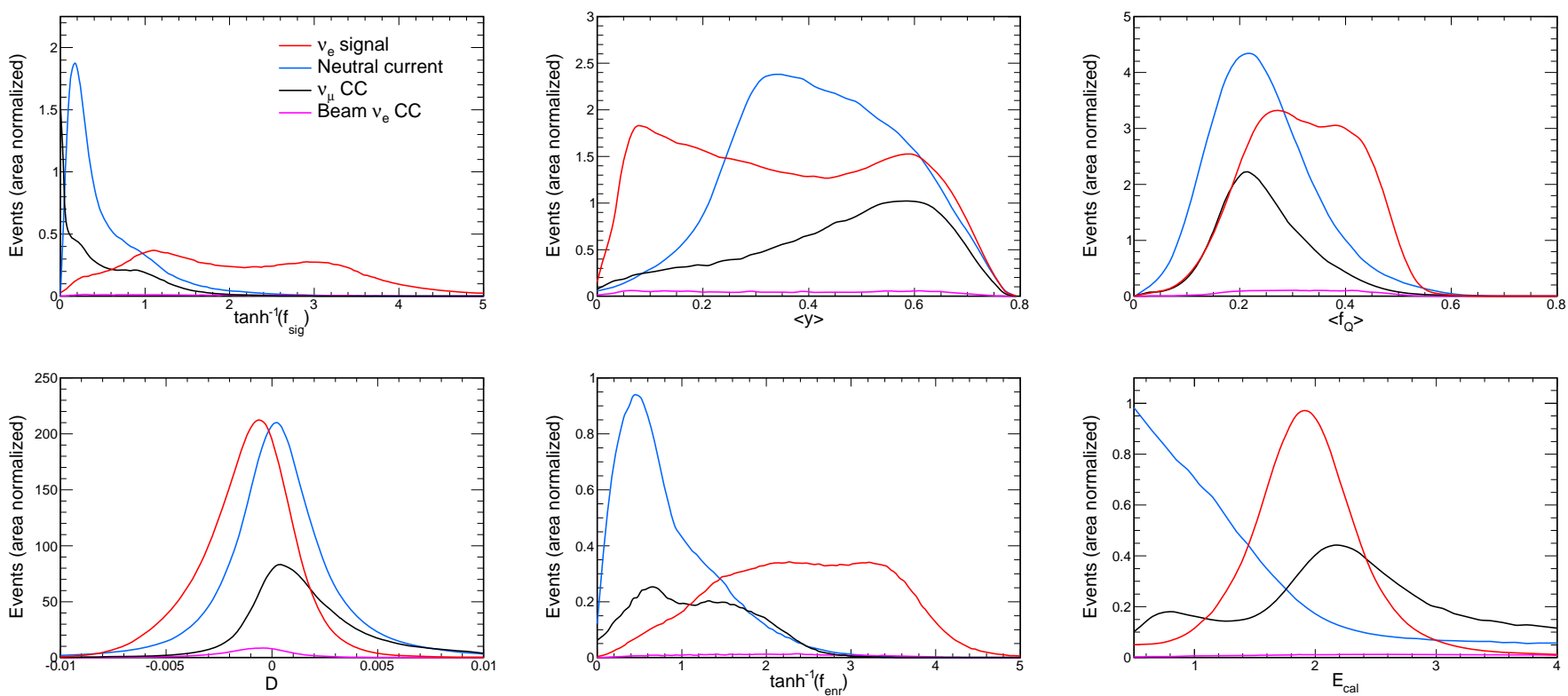

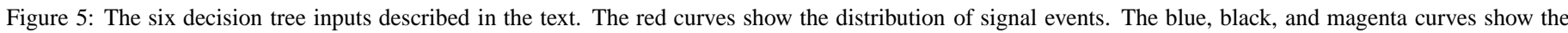

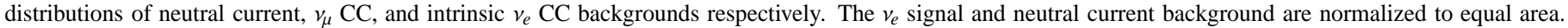

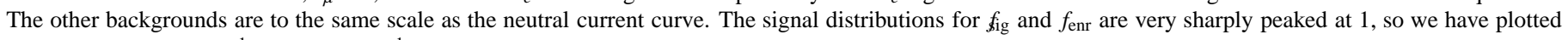
these quantities as $\tanh ^{-1}\left(f_{\text {sig }}\right)$ and $\tanh ^{-1}\left(f_{\text {enr }}\right)$ to keep the signal and background curves visible on the same vertical scale.

\subsection{Match energy difference}

This quantity measures whether the signal or background ${ }_{403}^{402}$ matches are the better matches on average. It is the difference of the weighted mean energy of each class of matches:

$$
D=\frac{\sum_{n, \operatorname{sig}} w_{n} E_{n}}{\sum_{n, \operatorname{sig}} w_{n}}-\frac{\sum_{n, \mathrm{bkg}} w_{n} E_{n}}{\sum_{n, \mathrm{bkg}} w_{n}}
$$

\subsection{Enriched fraction}

The final match list quantity, similar in construction to $f_{\text {sig }}$, is the weighted fraction of signal matches present among the signal and $\pi^{0}$-enriched matches (i.e., excluding the non-enriched ${ }^{411}$ background),

$$
f_{\text {enr }}=\frac{\sum_{n, \text { sig }} w_{n}}{\sum_{n, \text { enr }} w_{n}+\sum_{n, \text { sig }} w_{n}} .
$$

\subsection{Total calorimetric energy}

$\mathrm{NC}$ backgrounds skew heavily to low visible energy thanks ${ }^{418}$ to the energy removed by the exiting neutrino. The sum of all depositions $\left\{a_{i}\right\}$ recorded in the trial event,

$$
E_{\mathrm{cal}}=\sum_{i}^{\text {cells }} a_{i}
$$

is included as a final input so that the classifier knows the prior expectations of signal and background.

\subsection{Choice of a decision tree, and figure of merit}

There are many multivariate techniques capable of combining these six input quantities into a single classifier output. We investigated artificial neural networks, support vector machines, and decisions trees. An ensemble decision tree yielded the best performance of the approaches tried. One problem with other techniques is that the figure of merit (f.o.m.) that, for example, artificial neural network training aims to minimize is the meansquared-error of the classifier variable $c$ :

$$
\text { f.o.m. }=\sum_{i}^{\text {sig }}(1-c)^{2}+\sum_{i}^{\mathrm{bkg}} c^{2},
$$

where the sums run over the signal and background training samples. However, the figure of merit relevant to an experiment measuring the magnitude of a signal excess $s$ over a background $b$ with Poisson fluctuations is

$$
\text { f.o.m. }=\frac{s}{\sqrt{s+b}} .
$$

If events are binned according to, say, the classifier output, the generalization is simply to sum in quadrature the significances in the individual bins:

$$
\text { f.o.m. }=\sqrt{\sum_{i}^{\text {bins }} \frac{s_{i}^{2}}{s_{i}+b_{i}}} .
$$

While training a decision tree classifier, if the sample is divided at each step into subsamples 1 and 2 so as to maximize

$$
\frac{s_{1}^{2}}{s_{1}+b_{1}}+\frac{s_{2}^{2}}{s_{2}+b_{2}},
$$




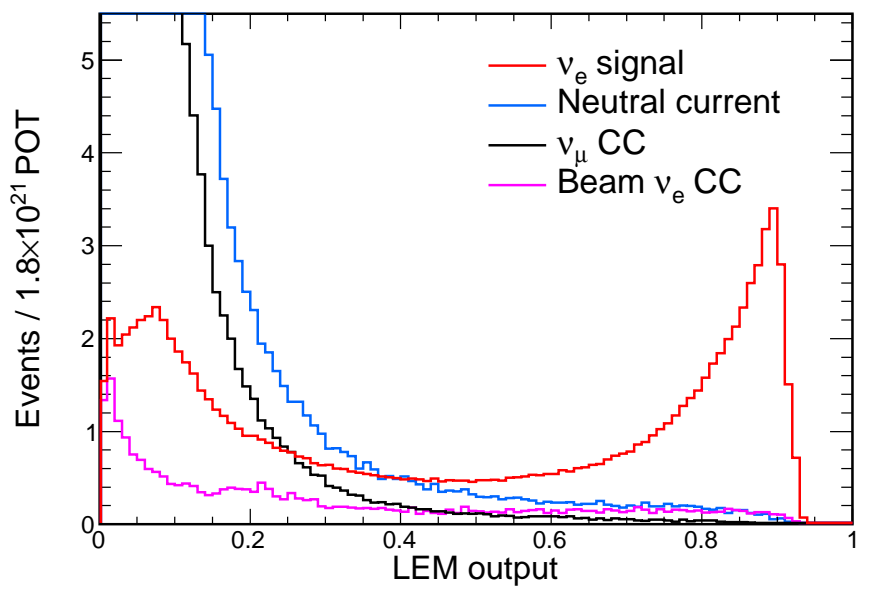

Figure 6: The distribution of the LEM output variable for $v_{e} \mathrm{CC}$ signal events (red) compared to the background components: neutral current (blue), $v_{\mu} \mathrm{CC}$ (black) and intrinsic beam $v_{e} \mathrm{CC}$ (magenta). In order to make the details in the signal-like region visible, the $y$-axis truncates much of the background peak. $95 \%$ of neutral current events and $98 \%$ of $v_{\mu}$ charged current events have LEM $<0.15$. The distributions are scaled to a nominal 3-year NuMI exposure [5] of $1.8 \times 10^{20}$ protons-on-target.

\section{Classification performance}

Figure 5 shows the distribution of the six input variables for ${ }^{467}$ all event classes in the $\mathrm{NO} v \mathrm{~A} v_{\mu} \rightarrow v_{e}$ analysis. Figure 6 shows $s^{468}$ the final LEM classifier output. Figure 7 shows the signal effi-469 ciency and purity obtained with various cuts on the LEM out-470 put. All curves come from Monte Carlo simulation of the ex-471 pected NOvA data set. We choose the cut on the LEM output ${ }^{472}$ variable that maximizes the figure-of-merit in Eq. (28). When ${ }^{473}$ applying LEM in a full experimental setting, one can fit the out-474 put distribution to gain additional discrimination power. $\quad 475$

Table 1 shows the expected number of signal and background ${ }^{476}$ events selected by the optimum LEM cut. The signal efficiency ${ }^{477}$ is $55 \%$ for a background mis-identification rate of $2.0 \%$. The $e^{478}$ muon track of $v_{\mu}$ CC events keeps their mis-identification rate ${ }^{479}$ particulary low. Background beam $v_{e}$ events are selected with a ${ }^{480}$ lower efficiency than signal $v_{e}$ events. This is possible due to the different underlying energy spectra of the two classes. As there ${ }^{481}$ is no absolute metric by which to judge the performance of the 482 LEM classification algorithm described here, we note simply483 that the performance shown is excellent for the physics goals of ${ }_{484}$ $\mathrm{NO} v \mathrm{~A}[5]$.

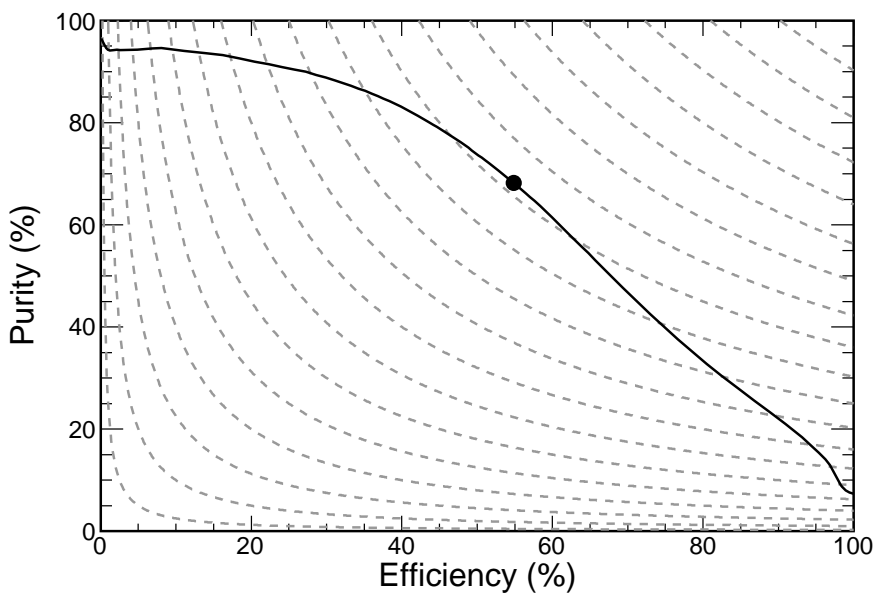

Figure 7: Efficiency and purity of the $v_{e}$ candidate sample selected by LEM for different cut positions. The dashed lines are curves of constant f.o.m. = $s / \sqrt{s+b}$, and the solid circle indicates the result of the optimum cut.

\section{Computational optimization}

\subsection{Speed}

While each individual energy calculation can be performed very quickly, classifying a single event takes some time given the large size of the library. For the NOvA application, a single event must be treated in a second or so, which is the time scale required by other steps already performed during $\mathrm{NO} v \mathrm{~A}$ event processing. Without specialized hardware to run the inner loop, techniques to manage the LEM matching time focus on reducing the number of energies that need to be calculated.

We achieve a significant speed-up by introducing a library "index". If trial event $A$ matches well to library event $B, A$ will likely match well to other library events that are, themselves, good matches to $B$. Similarly, if $A$ and $B$ match poorly, then $A$ will likely match poorly to library events similar to $B$.

A library index is formed by drawing 10,000 events uniformly from the full library and matching each of these to the full library. For each index event, a list of its 1,000,000 bestmatched library events is saved. This process happens ahead of time, at library creation. When a trial event is classified, it is compared first to the 10,000 index events to find the single bestmatching index event. The trial event is then compared only to the $1 \mathrm{M}$ sibling events of that index event, reducing the total number of energies calculated per trial event from 77,000,000 to $1,010,000$ - a significant speed improvement that takes the per trial matching time from $97 \mathrm{~s}$ down to $1.7 \mathrm{~s}$ on a $2.3 \mathrm{GHz}$ AMD Opteron processor. Empirically, we find that $85 \%$ of the trial event's "true" one-thousand top matches are captured with this indexed approached, and we find no noticeable degradation in the physics performance.

\subsection{Memory}

The speed optimization above is what allows the use of a $77 \mathrm{M}$ event library. However, such a large library strains memory resources. The full library is too large ( $\sim 53 \mathrm{~GB}$ each for the library and index) to read from disk for each event, yet it 


\begin{tabular}{lcc|ccc} 
& $v_{e}$ signal & Tot. bkgd. & $\mathrm{NC}$ & $v_{\mu} \mathrm{CC}$ & Beam $v_{e} \mathrm{CC}$ \\
\hline No selection & 105 & 1332 & 734 & 573 & 25 \\
LEM & 58 & 27 & 14 & 4.6 & 7.9 \\
\hline Efficiency & $55 \%$ & $2.0 \%$ & $2.0 \%$ & $0.8 \%$ & $32 \%$
\end{tabular}

Table 1: Number of events expected in each event category initially and again after an optimal LEM cut assuming a nominal 3-year NuMI exposure of $1.8 \times 1 \beta^{1}$ protons-on-target. The background is shown both as a total as well as broken down into $\mathrm{NC}, \nu_{\mu} \mathrm{CC}$, and intrinsic beam $v_{e} \mathrm{CC}$ components. The bottom row shows the efficiencies for selecting events in each category. The "no selection" row and the efficiencies derived from it count only those events with reconstructed visible energy between $0.5 \mathrm{GeV}$ and $4 \mathrm{GeV}$.

is larger than the typical per-core memory allocation on grid $_{527}$ computing nodes.

Thus, the library is converted from its original high-level for-529 mat into the memory representation used by a running job. This 530 representation includes the self-energy of each event. The con-531 version inflates the library slightly to $131 \mathrm{~GB}$, but the advantage is that it can now be shared between running processes. Each parallel matching job uses the mmap () system call to make the ${ }^{532}$ contents of this file visible in its address space. The mapping is marked read-only, so the kernel shares the pages between all the 533 running processes. For example, on a 64-core server, the mem-534 ory requirement to run 64 matching jobs is still only $131 \mathrm{~GB}, 535$ equivalent to an unshared 2 GB per core. In case of memory 536 pressure, the kernel will discard pages, knowing that they can be retrieved from disk (that is, the library file essentially acts as swap space) although this will significantly impact perfor-537 mance.

\section{Other information available in the match list}

In addition to signal-or-background classification, the detailed truth information available in the list of best matches al-541 lows other information about the trial event to be inferred. One ${ }^{542}$ could extract probabilities for different interaction modes, the ${ }^{543}$ inelasticity, and so on, without requiring any independent re-544 construction. An application that has been pursued is the estimation of the incident neutrino energy for $v_{e}$ CC events. Simply ${ }_{545}$ by averaging the true neutrino energies of the best signal library matches and calibrating the resulting estimator, we achieve an energy resolution of $8.8 \%$ on signal events selected by the os-546 cillation analysis, competitive with other energy estimators in $n_{547}$ $\mathrm{NO} v \mathrm{~A}$.

\section{Summary}

The Library Event Matching algorithm compares input trial 553 events to a large library of known events using all the infor-554 mation available, making LEM an optimal classifier given a555 sufficiently large library. The $\mathrm{NO} v \mathrm{~A}$ implementation of $\mathrm{LEM}_{556}$ has demonstrated excellent performance in separating $v_{e}$ sig-557 nal from the key backgrounds, and a few simple optimizations have maintained practical computational requirements despite the large number of library events used. Within the NOvA context, the LEM technique has potential applications from recon- 559 struction of the hadronic system to the event energy measure 560 described above. More broadly, LEM can be applied to completely different particle detectors or imaging systems in an array of fields and industries, wherever one needs to classify finegrained images of objects whose visual characteristics vary in known ways.

\section{Acknowledgments}

The authors thank the NOvA collaboration for use of its Monte Carlo simulation software and related tools. This work was supported by the the US DOE under award DESC0006543.

\section{Appendix A. Additional technical notes}

A few technical notes are included in this Appendix so as not to break up the discussion in the main text.

\section{Appendix A.1. Relation to other classification techniques}

If $f_{\text {sig }}$ and $f_{\text {enr }}$ were calculated unweighted, then those variables would be $k$-nearest-neighbors classifiers, albeit with very large input vectors. With the weights $w_{n}$ applied, they act as kernel density estimators. Note that

$$
\sqrt{2 E}=\sqrt{\sum_{i j}\left(a_{i}^{\beta}-b_{i}^{\beta}\right) T_{i j}\left(a_{j}^{\beta}-b_{j}^{\beta}\right)}
$$

is a metric for the space of possible event images. That is, distances defined in this way obey the triangle inequality. For a Gaussian kernel in this space one would expect $w_{n} \sim \exp (-E)$, which contrasts with the optimal value of $\gamma=10$ found in practice. Similarly $\langle y\rangle$ is an estimator for the true value of $y$ using the same kernel.

Methods exist to efficiently find nearest-neighbors in general metric spaces without having to rely on heuristics such as the library index in Section 7.1. Testing of a vantage-point tree [10] indicated its performance was affected by the curse of dimensionality. A large fraction of the nodes would have to be entered during a typical search.

\section{Appendix A.2. Energy calculation when $r_{i j}=0$}

The transfer matrix element $T_{i j}$ as written in Eq. (9) diverges when $i=j$ since $\Delta p_{i i}$ and $\Delta c_{i i}$ are zero. Thus, for nearby 
cell pairs $\left(\Delta p_{i j} \leq 5\right.$ and $\left.\Delta c_{i j} \leq 5\right)$, the energy calculation is per-598 formed as if the charge is distributed uniformly over each cell, ${ }^{599}$ with

$$
T_{i j}=\int_{0}^{1} \int_{0}^{1} \int_{0}^{1} \int_{0}^{1}\left[r_{i j}(x, y, u, v)\right]^{-\alpha} d x d y d u d v,
$$

where $(x, y)$ and $(u, v)$ scan over the areas of cells $i$ and $j$ and where $r_{i j}$ here is a generalization of the discrete distance used in the main text:

$$
r_{i j}(x, y, u, v)=\sqrt{\left(\frac{\Delta p_{i j}+x-u}{\sigma_{p}}\right)^{2}+\left(\frac{\Delta c_{i j}+y-v}{\sigma_{c}}\right)^{2}} .
$$

For more distant pairs the simplified form of the transfer matrix given in Eq. (9) is sufficient.

\section{Appendix A.3. Neutrino oscillation weights}

The retained matches are weighted according to Eq. (19), which includes the probability for flavor oscillation. The probabilities used are

$$
\begin{aligned}
& P\left(v_{\mu} \rightarrow v_{e}\right)=\sin ^{2} \theta_{23} \sin ^{2} 2 \theta_{13} \sin ^{2}\left(\frac{1.27 \Delta m^{2} L}{E}\right) \\
& P\left(v_{e} \rightarrow v_{\mu}\right)=\sin ^{2} \theta_{23} \sin ^{2} 2 \theta_{13} \sin ^{2}\left(\frac{1.27 \Delta m^{2} L}{E}\right) \\
& P\left(v_{\mu} \rightarrow v_{\mu}\right)=1-\sin ^{2} 2 \theta_{23} \sin ^{2}\left(\frac{1.27 \Delta m^{2} L}{E}\right) \\
& P\left(v_{e} \rightarrow v_{e}\right)=0
\end{aligned}
$$

where $L=810 \mathrm{~km}$ is the oscillation baseline, $E$ is the neutrino energy in $\mathrm{GeV}$, and the oscillation parameters are taken to be

$$
\begin{aligned}
\theta_{13} & =9.2^{\circ} \\
\theta_{23} & =38.5^{\circ} \\
\Delta m^{2} & =2.35 \times 10^{-3} \mathrm{eV}^{2} .
\end{aligned}
$$

These oscillation probabilities are first-order approximations to the full expressions. This is both for practical reasons - the second-order effects are poorly determined and are in fact what $\mathrm{NO} v \mathrm{~A}$ aims to measure - and because there is no requirement for the library have any particular distribution of events in it. The second order effects can pull the probabilities higher or lower, making this weighting a reasonable middle ground for the library. The library is also made devoid of intrinsic $v_{e}$ from the NuMI beam by setting that survival probability to zero. The overall prefactor on the $v_{\mu} \rightarrow v_{e}$ (signal) line relative to the background lines actually does not enter in practice since the signal, background, and $\pi^{0}$-enriched background classes are scaled to have equal total weight in the library.

[1] P. Adamson et al. (MINOS), Phys. Rev. Lett. 107, 181802 (2011).

[2] P. Adamson et al. (MINOS), Phys. Rev. Lett. 110, 171801 (2013).

[3] J. P. Ochoa, Ph.D. thesis, California Institute of Technology, FERMILABTHESIS-2009-44 (2009).

[4] R. Toner, Ph.D. thesis, University of Cambridge, FERMILAB-THESIS2011-53 (2011)

[5] D. S. Ayres et al. (NOvA), FERMILAB-DESIGN-2007-01 (2007); R. B. Patterson, for NOvA, Nucl. Phys. Proc. Suppl. 235-236, 151 (2013).
[6] K. Anderson et al., FERMILAB-DESIGN-1998-01 (1998).

[7] A. Ferrari, P. R. Sala, A. Fasso, and J. Ranft, CERN-2005-10 (2005); G. Battistoni et al., AIP Conf. Proc. 896, 31 (2007).

[8] C. Andreopoulos, for GENIE, Acta Phys. Polon. B 40, 2461 (2009).

[9] S. Agostinelli et al., Nucl. Instrum. Meth. A 506, 250 (2003).

[10] J. Uhlmann, Inform. Process. Lett. 40, 4 (1991); P. N. Yianilos, Proceedings of the Fourth Annual ACM-SIAM Symposium on Discrete Algorithms, 311 (1993). 